\title{
Dehydroepiandrosterone can restore the function of the ovaries - a series of 5 cases and a review of the literature
}

\author{
Katarzyna Jankowska', Radosław Maksym², Wojciech Zgliczyński \\ 'Department of Endocrinology, Bielanski Hospital, Centre of Postgraduate Medical Education, Warsaw, Poland \\ 2Department of Reproductive Health, St. Sophia Hospital, Centre of Postgraduate Medical Education, Warsaw, Poland
}

\begin{abstract}
Introduction: We studied the impact of dehydroepiandrosterone (DHEA) supplementation in women with diminished ovarian reserve and very low DHEA-S in the blood.

Aim: In vivo study. The paper also presents a review of the literature regarding diminished ovarian reserve and the use of dehydroepiandrosterone.

Material and methods: The paper presents a description of 5 patients with diminished ovarian reserve (DOR). Patients reported because of problems with getting pregnant. Infertility lasted for several years. The patients disagreed on in vitro fertilization (IVF) for ethical and religious reasons. All of the presented patients were diagnosed with diminished ovarian reserve (very low anti-Müllerian hormone or high follicle stimulating hormone, elevated estradiol concentration on day 3 of the cycle). We also found a very low dehydroepiandrosterone sulphate (DHEA-S) concentration. The patients were given dehydroepiandrosterone. After several months of treatment (3-6 months), the patients became pregnant. None of them had procedures for in vitro fertilization.

Results: Five patients gave birth to healthy children. Our experience with DHEA is much greater, but these 5 cases are very well documented. The obtained results indicate that DHEA supplementation in conditions of its deficiency improves the function of the ovaries and increases the chance of pregnancy.

Conclusions: In some cases the aging of the ovaries can be delayed by administering DHEA. This effect occurs specially when the endogenous concentration of DHEA-S is reduced. In the case of reduced ovarian reserve, DHEA-S concentration should be determined.

Key words: dehydroepiandrosterone, diminished ovarian reserve, aging, infertility.
\end{abstract}

Corresponding author: Katarzyna Jankowska PhD, MD, Department of Endocrinology, Bielanski Hospital, Centre of Postgraduate Medical Education, 80 Cegłowska St, 01-809 Warsaw, Poland, phone: +48 691054 045,

e-mail: katarzynakamilajankowska@gmail.com 


\section{Introduction}

Moving the procreation plans to a later age is often associated with greater problems in obtaining the desired pregnancy.

Worsened ovarian function causes estrogen deficiency and lowering the ovarian reserve [1]. Hormonal insufficiency causes metabolic consequences such as premature atherosclerosis, a decrease in bone mineral density (osteopenia, osteoporosis), as well as urogenital symptoms: vaginal dryness and infections. The most important aspect for each woman is the reduction in fertility.

The response to ovarian hormone insufficiency is elevated follicle stimulating hormone (FSH) concentration on the $3^{\text {rd }}$ day of the cycle. Elevated estradiol concentrations ensure disturbed recruitment of the dominant follicle already at the beginning of the cycle. Although the cycles are still ovulatory (the dominant follicle can be seen in the ultrasound), pregnancy does not occur. This phenomenon is explained by the worse quality of follicles - that is, the concentration of hormones inside the follicle is lower [2]. Recently, it has also been proven that the aging of the ovary reduces the expression of FSH receptors and androgen receptors $[3,4]$.

In some patients, the dehydroepiandrosterone sulphate (DHEA-S) deficiency can sometimes be diagnosed. Only in the next sequence is there a full-blown estrogen deficiency (hot flushes, night sweats, osteopenia). It seems that androgen deficiency may be the first symptom of ovarian hormone deficiency.

In some women with infertility and low ovarian reserve, dehydroepiandrosterone (DHEA) may improve ovarian reserve. Probably this is not so effective if the concentration of endogenous DHEA is correct.

\section{Material and methods}

We present 5 cases of patients who could not get pregnant. They had been trying for a child for several years. They were treated with various medications by diverse specialists. The patients did not agree to artificial reproductive technology (ART) for ethical and religious reasons. The patients' age was 34-42 years (mean: 38 years), the time of applying for pregnancy was 2-6 years (mean: 4 years). Patients had elevated FSH and estradiol levels on the third day of the cycle and significantly reduced DHEA-S levels. One patient did not have elevated FSH due to partial hypopituitarism (2 years earlier she had surgery of the pituitary gland due to inactive adenoma). Administration of DHEA (25-50 mg) for several months (3-6 months) resulted in pregnancy. Our experience with the use of DHEA is much greater; however, in these 5 patients, pregnancy was clearly associated with the use of DHEA and is very well documented.

\section{Results}

The results were presented in Table I.

\section{Discussion}

The investigation by Casson et al. [5] might be the first to utilize DHEA in the assisted reproductive techniques mediated by improvement of the ovarian response. Several years later, Barad and Gleicher [6] described an astonishing case with rapidly increased oocyte production after DHEA supplementation. Thus, scientists were interested in the study of DHEA, since it may function as an anti-aging agent to rescue diminished ovarian reserve (DOR) $[5,6]$.

Our experience shows that these are clinical situations when the initially determined DHEA-S concentration is below the norm for sex and age. DHEA administration is specially effective for patients who have low endogenous DHEA-S levels in their blood.

\section{Ovarian reserve}

The following markers are used as predictors of ovarian reserve: $\mathrm{FSH}$ on the $3^{\text {rd }}$ day of the cycle, estradiol on the

Table I. Patients with infertility and DHEA-S below the reference age

\begin{tabular}{|c|c|c|c|c|c|c|c|c|c|}
\hline No. & Age & $\begin{array}{c}\text { Duration of } \\
\text { infertility } \\
\text { [years] }\end{array}$ & $\begin{array}{c}\text { FSH }-3^{\text {rd }} \\
\text { day of cycle } \\
{[\mathrm{IU} / \mathrm{ml}]}\end{array}$ & $\begin{array}{c}\text { AMH } \\
{[n g / m l]}\end{array}$ & $\begin{array}{c}\text { Estradiol } \\
3^{\text {rd }} \text { day of } \\
\text { cycle }\end{array}$ & $\begin{array}{l}\text { DHEA-S } \\
{[\mu \mathrm{g} / \mathrm{dl}]}\end{array}$ & $\begin{array}{c}\text { Testosterone } \\
\text { [ng/ml] }\end{array}$ & $\begin{array}{l}\text { Result of } \\
\text { treatment }\end{array}$ & $\begin{array}{l}\text { After what } \\
\text { time of } \\
\text { DHEA } \\
\text { application } \\
\text { did } \\
\text { pregnancy } \\
\text { occur? }\end{array}$ \\
\hline 1 & 42 & 6 & 29.9 & 0.28 & 72.8 & $\begin{array}{c}24.6 \\
\text { (N 25.9-460.2) }\end{array}$ & $\begin{array}{c}9 \\
\text { (N 14-76) }\end{array}$ & $\begin{array}{c}\text { Birth of } \\
\text { a healthy child }\end{array}$ & 3 months \\
\hline 2 & 35 & 5 & 14.65 & 1.7 & 106.8 & $\begin{array}{c}64.32 \\
\text { (N 98.8-340) }\end{array}$ & $\begin{array}{c}0.13 \\
\text { (N 0.06-0.82) }\end{array}$ & $\begin{array}{c}\text { Birth of } \\
\text { a healthy child }\end{array}$ & 4 months \\
\hline 3 & 38 & 2 & $\begin{array}{c}6.3 \text { - attention: } \\
\text { after pituitary } \\
\text { surgery }\end{array}$ & 2.1 & 50.5 & $\begin{array}{c}49 \\
\text { (N 58-227) }\end{array}$ & $\begin{array}{c}0.15 \\
(N 0.1-0.6)\end{array}$ & $\begin{array}{c}\text { Birth of } \\
\text { a healthy child }\end{array}$ & 5 months \\
\hline 4 & 34 & 5 & 20.23 & 0.46 & 49.4 & $\begin{array}{c}58.5 \\
\text { (N 25.9-460.2) }\end{array}$ & - & $\begin{array}{c}\text { Birth of } \\
\text { a healthy child }\end{array}$ & 5 months \\
\hline 5 & 34 & 3 & 7.39 & 0.2 & 40.1 & $\begin{array}{c}99.9 \\
\text { (N 25-460) }\end{array}$ & $\begin{array}{c}78 \\
\text { (N 14-76) }\end{array}$ & $\begin{array}{c}\text { Birth of } \\
\text { a healthy child }\end{array}$ & 6 months \\
\hline
\end{tabular}


$3^{\text {rd }}$ day of the cycle, inhibin B and anti-Müllerian hormone $(\mathrm{AMH})$ - regardless of the day of the cycle, and antral follicle count (AFC) in transvaginal ultrasound.

\section{Clinical picture}

Usually a 30-35-year-old woman, applying for pregnan$\mathrm{cy}$, reports to a doctor. Menstruation is usually regular, but there may be shorter cycles (23-25 days) or longer breaks (35-60 days). There may be mild exacerbation of symptoms: a feeling of heat and increased sweating.

Hormonal examination shows hypergonadotropic hypogonadism (i.e. primary hypogonadism). In hormonal tests, elevated levels of FSH (FSH > 15-20 IU/l), high concentrations of estradiol $(\mathrm{E} 2>50-80 \mathrm{pg} / \mathrm{ml})$ on day 3 of the cycle, and decreased estradiol concentration in the second phase of the cycle are observed. Ovarian reserve, i.e. AMH $<0.5 \mathrm{ng} / \mathrm{ml}(<1 \mathrm{ng} / \mathrm{ml})$ and inhibin $\mathrm{B}$, are very low $[7,8]$.

The concentration of FSH $>40 \mathrm{IU} / \mathrm{l}$ found at least twice in the 4-6 weeks interval entitles one to recognize premature ovarian failure.

The greater the hormonal insufficiency of the ovaries, the higher is the FSH concentration. Low estradiol levels are caused by ovarian failure and it stimulates the pituitary gland to produce a gonadotropic hormone (high FSH) by feedback.

Premature ovarian failure (POF) is considered to be the end of ovarian function before 40 . This term is understood as the ending of hormonal and generative ovarian function caused by depletion of the ovarian follicle pool before the age typical of physiological menopause - that usually is around 50-51 years old.

Follicle stimulating hormone is a glycoprotein pituitary hormone and the main regulator of the reproductive system [9]. It acts on the cells of the granular layer of vesicles in the ovaries of women and - let us not forget - on Sertoli cells in the testes of men. FSH works through membrane receptors (FSHR). The expression of FSHR is also described in blood monocytes, osteoclasts and adrenal glands. It was also found that FSH increases the secretion of vascular endothelial growth factor (VEGF), leading to increased angiogenesis.

It was also established that FSH mediates the growth of cells and neoangiogenesis, that is enhanced by FSHR overexpression in vessels of tumors. For example, the positive effects of degarelix (a gonadoliberin receptor antagonist) in the treatment of colon cancer are considered to be the result of a decrease in FSH levels $[4,10]$.

Anti-Müllerian hormone is a glycoprotein produced by the granular cells of antral follicles in the ovary. Anti-Müllerian hormone belongs to the peptide growth and differentiation factors. Anti-Müllerian hormone level does not depend on the day of the cycle. Its level decreases with women's age, and is considered as a good marker of fertility [11]. In patients with polycystic ovary syndrome (PCOS) Anti-Müllerian hormone levels are significantly elevated, because this syndrome has an excessive number of ovarian follicles (but not ripe). Typically, the concentration of AMH in the PCO syndrome is greater than $5.5 \mathrm{ng} / \mathrm{ml}$.
Inhibin B also serves to assess ovarian reserve, but its determination depends on the phase of the cycle. It is produced by granular cells of early antral follicles, primarily during the follicular phase of the menstrual cycle. The concentration of inhibin B in the early follicular phase reflects the number and quality of ovarian follicles. The fewer follicles, the lower is the inhibin B level [12].

\section{Why does DHEA improve diminished ovarian reserve?}

Ovarian aging is usually caused by changes resulting from the aging of the organism such as incorrect vascularization of the vesicles (reduction of the amount of oxygen that reaches the vesicle through the vessels), oxidative stress, toxic compounds, infections, and genetic mutations [13]. It has also been proven that ovarian reserve is much more depleted in women who smoke cigarettes. They will experience menopause 2-3 years earlier than nonsmokers.

Studies suggest that DHEA supplementation in some women with reduced ovarian reserve may lead to a natural pregnancy. DHEA supplementation in such patients has been shown to increase the number of preantral follicles and small antral follicles. It also improves the number of embryos obtained in embryo culture in vitro $[14,15]$.

DHEA is then used with the hope of improving low ovarian reserve in cases of elevated FSH, decreased AMH, poor response to stimulation, low amount of antral follicles in the ovaries, and advanced patient age [16].

The mechanism of action of DHEA in ovaries is not fully understood $[17,18]$. Recent studies have shown that administration of DHEA increases the expression of androgen receptors (AR) as well as FSHR on granular cells in the ovaries. Through AR or metabolic pathways, androgens are believed to promote recruitment and initiation of primordial follicle growth; stimulate development of primary, preantral, and antral follicles [18]; rescue follicles from atresia; suppress apoptosis [19, 20]; and upregulate FSH expression receptor [21-23].

A well-known fact is that ovary insufficiency causes an increase in FSH concentration. Nevertheless, even at high FSH levels, folliculotropin has no biological effect (recruitment of the follicles and ovulation). This is - as we now know - because the FSH receptors are no longer sensitive to FSH $[24,25]$.

\section{Low AMH? High FSH? Mark DHEA-S!}

If the ovarian reserve is lowered, high doses of gonadotropins (FSH) are used for stimulation. Instead of giving increasing doses of FSH to stimulate ovulation, the DHEA-S concentration should be defined [4, 26, 27].

Dehydroepiandrosterone is a steroid hormone from androgen family. It is made from cholesterol by the adrenal glands and in a much smaller amount by the ovaries and testicles. DHEA is produced by adrenal zona reticularis (50\%) and ovarian theca cells (20\%), and also derived from circulating DHEA-S (30\%) [28]. DHEA levels steadily decrease with age (by $10 \%$ per decade), reaching a nadir after the age of 80 years [29], suggesting that DHEA may be involved in the aging process. DHEA acts anabolically and is a precursor to androgens and estrogens.In women androgens are made 
from DHEA (mainly testosterone). In men with DHEA, mainly estrogens (estradiol and estrone) are formed. In turn, DHEA-S, i.e. DHEA-SO, is a hormone formed from DHEA.

DHEA production begins at the age of 6-8 years and reaches the maximum level at the age of about 25-30 years, and then begins to decline, to reach the level from the period preceding adolescence at 70 years of age. This decrease is further aggravated by old-age diseases, for example cardiovascular diseases, cancers, immunological deficiencies, depression and diabetes [30].

DHEA works through receptors for androgens and estrogens. Androgenic action is a consequence of peripheral DHEA conversion via androstenedione to testosterone. It is also a substrate for the synthesis of estrogen in subcutaneous fat tissue, fat tissue of mammary glands, hair follicles and other tissues. DHEA supplementation does not inhibit the secretion of endogenous DHEA (Figure 1).

A reduced level of DHEA and DHEA- $\mathrm{SO}_{4}$ may indicate [31]: adrenal insufficiency, e.g. Addison's disease (primary adrenal insufficiency); hypopituitarism; hypothyroidism; autoimmune diseases [32, 33].

The effects on DHEA and DHEA-SO $\mathrm{S}_{4}$ decline in the body also have long-lasting stress, which is sometimes referred to in the American literature as "adrenal insufficiency syndrome".

Elevated levels of DHEA and DHEA-SO ${ }_{4}$ may indicate: adrenocortical tumor; adrenal cancer; overgrow the adrenal cortex; polycystic ovary syndrome; about ACTH stimulation, or adrenocorticotropic hormone.

The impact of DHEA decline on the aging process and age-related diseases.

Reduction in DHEA with age is of clinical significance and is associated with various age-related illnesses. The pos- itive relationship between DHEA concentration and muscle mass, muscular strength as well as mobility and lower risk of falls in the elderly has been proven.

In addition, DHEA has a positive effect on bone mineral density (BMD) through conversion to estrogens (human in vitro osteoblasts exhibit aromatase activity), but also directly through mitogen-activated kinase signaling pathways. DHEA concentrations show a positive correlation with BMD both in women and men.

Regarding neuropsychiatric diseases, the relationship between DHEA and cognitive impairment is still being investigated. There is a clear relationship between DHEA levels and mood disorders [34]. It was found that low DHEA concentrations were associated with symptoms of depression.

Research is ongoing on the relationship between DHEA and risk factors for cardiovascular disease such as hypercholesterolemia and carbohydrate intolerance. Studies have shown that low DHEA levels are associated with a higher risk of atherosclerosis, heart failure, cardiovascular complications, and overall mortality.

DHEA plays an important role in sexual life for both sexes. Low levels of DHEA are associated with a higher risk of erectile dysfunction in men and low sexual activity in women.

\section{Review}

\section{First case description of premature ovarian insufficiency}

Premature ovarian insufficiency (POI) was defined by de Moraes-Ruehsen and Jones in 1967 as a nonphysiological cessation of menstruation before 40 years after puberty [35]. In

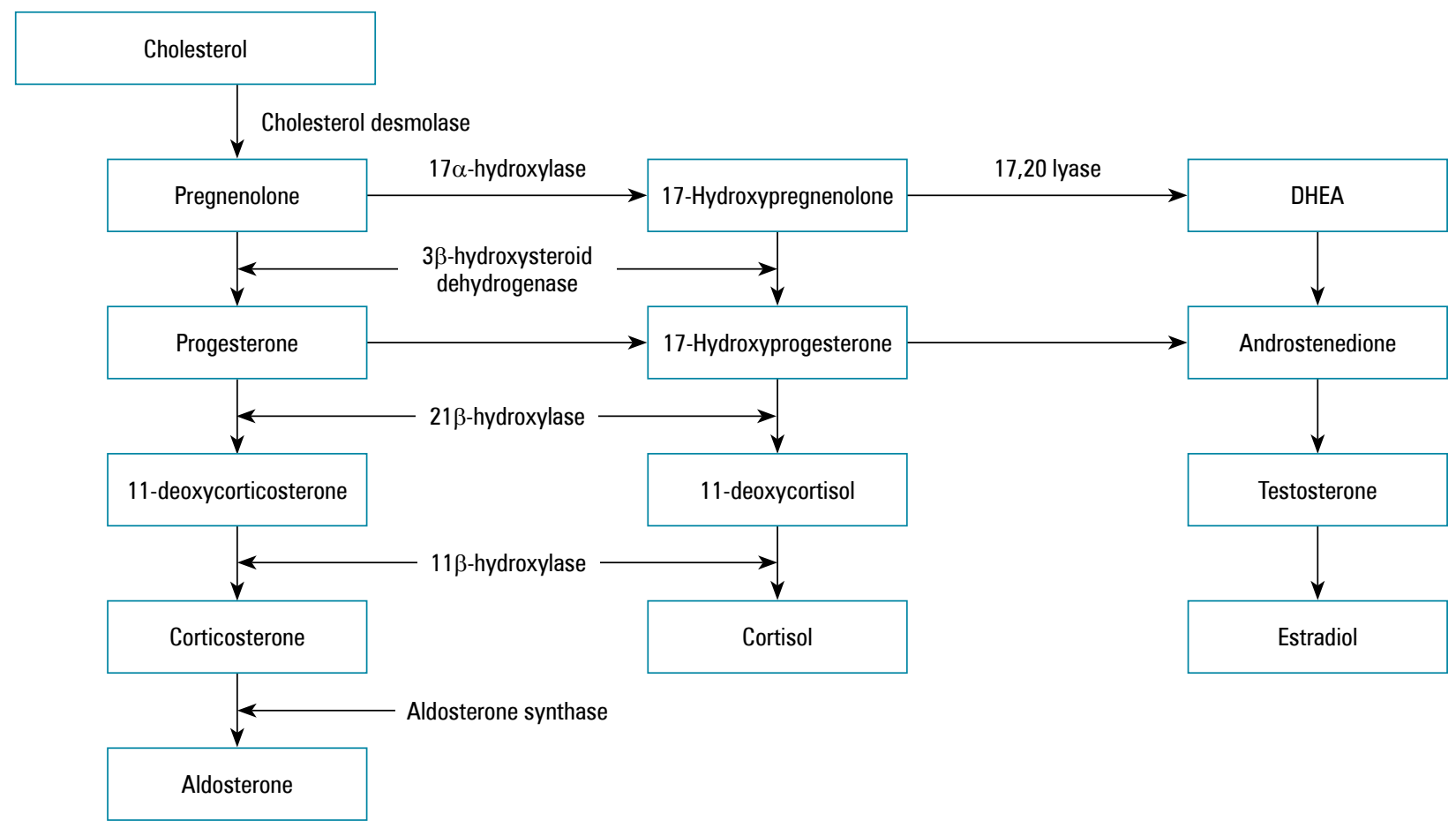

Figure 1. Steroidogenesis (modified from: Miller WL, Auchus RJ. The molecular biology, biochemistry, and physiology of human steroidogenesis and its disorders. Endocr Rev 2011; 32: 81-151) 
1939, the hormone profile in women with POF was described as hypergonadotropic hypoestrogenism. The clinical picture of POI was presented in detail in 1950 by Atria [36]. This author described 20 young women before 35 with secondary amenorrhea, hot flushes, infertility and atrophic endometrium.

\section{Decreased DHEA in low ovarian reserve}

A recent meta-analysis of 14 studies showed that women with POI are characterized by lower levels of androgens that healthy controls: testosterone, dehydroepiandrostenedione sulfate, androstenedione [37]. Others estimated that low DHEA-S levels are quite common and were described in $65 \%$ women with DOR [32], and are much frequent than other endocrinopathies and immunopathies. Although androgen levels are lower than in women with regular cycles, after adjustment for age there is no difference between women with POI and natural post-menopausal women in levels of testosterone, androstenedione and DHEA-S [30]. Some authors report no relation between serum DHEA-S levels with oocyte production and pregnancy rates [38]. Autoimmunity can play a role in the mechanism of DHEA action on DOR. Occurrence of anti-thyroid antibodies in women with POI was associated with enhanced reduction of DHEA-S. Administration of DHEA resulted in decrease in thyroperoxidase autoantibodies levels [33].

In a group of 25 patients with POF a high proportion ( $44 \%$ vs. $4 \%$ ) of patients with autoantibodies was described. Interestingly, once again autoimmunity was associated with higher DHEA-S suppression [31].

\section{Supplementation of DHEA in IVF}

Diminished ovarian reserve is a major challenge for ART, because of the growing incidence and significant decrease in the effectiveness of stimulation and outcome of IVF, due to decreased ovarian reserve and oocyte quality. However, ESHRE (European Society of Human Reproduction and Embryology) guidelines do not support any treatment modality in premature ovarian insufficiency except oocyte donation and indicate that androgen supplementation has limited evidence [39]. Many attempts are made to increase the effectiveness of treatment in the group of infertile DOR patients. DHEA supplementation is one of the approaches to the problem. A recent meta-analysis showed that the clinical pregnancy rates were increased significantly with DHEA pre-treatment (OR $=1.47,95 \%$ CI: $1.09-1.99$ ). There were no differences in the number of oocytes retrieved, the IVF cancellation rate, or the miscarriage rate [40].

An older meta-analysis showed DHEA as a therapy able to improve IVF outcomes in women with diminished ovarian reserve. However, randomized controlled trials did not show the benefit of DHEA therapy, although one prospective randomized study revealed that DHEA can increase fertility potential in women without diminished ovarian reserve [41]. Since patients with normal reserve when treated with DHEA had a significantly higher live birth rate and a lower abortion rate [42], it was suggested that all women above 35 years of age could be supplemented with DHEA to reduce the miscarriage rate [43].
The mechanism underlying beneficial activity of DHEA was found to be complex. DHEA supplementation increases the number of top-quality embryos, transferred embryos and fertilization rate compared with those without DHEA supplementation. Supplementation with DHEA was shown to reduce the DNA damage and apoptosis rate, and enhance the mitochondrial mass and mitochondrial dehydrogenase activity in cumulus cells [44]. After DHEA supplementation a higher embryo score is accompanied by changes in follicular fluid composition that includes bone morphogenic protein-15 (BMP-15) [45]. Others showed that the beneficial effect of DHEA on the ovary is related to a reduced level of a senescence marker, namely senescence-associated $\beta$-galactosidase (SA- $\beta$-gal) in cumulus cells, as well as in a granulosa cell line [46]. Substitution also upregulated the expression of AR and FSHR on granulosa cells and higher upregulation was associated with better stimulation outcomes [47]. DHEA and its downstream products may also act through modification of the immune response. The supplementation can also enhance the Th1 immune response and switch the balance of the Th1/Th2 response. DHEA treatment can increase $\mathrm{T}$ lymphocyte infiltration in mice, resulting in a decline in the CD4+ T lymphocyte population, upregulation of the CD8+ T lymphocyte population and changing of the balance between CD4+/CD8+ T cells [48].

The levels of DHEA-S and testosterone were proposed as predictive factors of the IVF success rate in DOR patients. Basal levels of DHEA-S and testosterone were similar between pregnant and non-pregnant cases. Basal levels of androgens, in contrast to AMH and FSH levels, do not predict the outcome parameters of the IVF procedure [19].

\section{Supplementation of DHEA in natural reproduction}

Supplementation of DHEA was also applied in infertile women with low ovarian reserve trying to conceive spontaneously. One of the preliminary case series, by Mamas and Mamas, reports five women with POI treated with DHEA. The therapy caused reduction in FSH levels and spontaneous conception in all patients within 1-6 months [20]. DHEA was proven to significantly increase $\mathrm{AMH}$ in women with DOR. Beneficial results were found in AFC, estradiol, inhibin B and FSH levels [12, 49, 50]. Studies on women pre-treated with DHEA, because of poor response, showed a surprisingly high proportion of spontaneous incidental pregnancies and ongoing pregnancies at the level of $21.05 \%$ and $13.15 \%$ respectively. A recent study showed that only 2 out of 20 treated women conceived spontaneously in the DHEA group [51].

In the rat model of DOR administration of DHEA partially reverses the phenotype and reduces the atresia rate of the follicles. After supplementation animals had a significantly higher number of primordial, primary, and growing follicles than untreated animals. Despite the androgen supplementation the number of follicles was still lower than in control rats without DOR [52].

A study performed in a rat model of DOR revealed that there is a therapeutic window for DHEA supplementation. Higher doses of DHEA do not improve ovarian reserve and 
pregnancy outcome, but induce PCOS-like morphology of ovaries with impaired fertility. It indicates the need of personalization of the treatment. Adjustment of a proper dose of DHEA and the selection of patients who will benefit from the therapy would increase the effectiveness [53].

\section{Conclusions}

The paper presents 5 cases of patients with reduced ovarian reserve who became pregnant and gave birth to healthy children after administering DHEA. The literature also describes spontaneous pregnancy incidents in infertile patients with reduced ovarian reserve who received DHEA. Similarly, some patients qualified for the IVF procedure using the donor's egg cells were given pregnancies before the procedure of in vitro fertilization began. It has been found that the administration of DHEA in patients with premature ovarian failure prolongs the chances of pregnancy, reduces the risk of miscarriage and increases the chance of success when using IVF methods [14, 15, 16, 54-57].

In women with infertility and low ovarian reserve, DHEA may improve ovarian reserve. Presented case reports confirm this effect of DHEA. Based on previous studies, it is known that DHEA stimulates the maturation of primordial follicles to preantral vesicles. Recently, this effect has been shown to increase the expression of androgen receptors and receptors for FSH in the ovaries [4]. According to the authors, it is specially effective to administer this hormone to women who have low endogenous secretion of dehydroepiandrosterone. DHEA-S concentration in the blood should be measured in all women with diminished ovarian reserve, especially those who are qualified for ART. The question to consider is whether DHEA-S levels should be measured in the blood of infertile men. There are FSH receptors on Sertoli cells [4]. If DHEA-S increases the expression of FSH receptors and sensitivity to FSH, a deficiency of this hormone may interfere with the process of spermatogenesis. It would be interesting to study the levels of FSH, inhibin B and DHEA-S in infertile men. Perhaps DHEA-S deficiency disrupts sperm maturation.

\section{Conflict of interest}

The authors declare no conflict of interest.

\section{References}

1. Lin LT, Cheng JT, Wang PH, Li CJ, Tsui KH. Dehydroepiandrosterone as a potential agent to slow down ovarian aging. J Obstet Gynaecol Res 2017; 43: 1855-62.

2. Yeung TW, Chai J, Li RH, Lee VC, Ho PC, Ng EH. A randomized, controlled, pilot trial on the effect of dehydroepiandrosterone on ovarian response markers, ovarian response, and in vitro fertilization outcomes in poor responders. Fertil Steril 2014; 102: 108-15.

3. Shiina $\mathrm{H}$, Matsumoto $\mathrm{T}$, Sato $\mathrm{T}$, et al. Premature ovarian failure in androgen receptor-deficient mice. Proc Natl Acad Sci USA 2006; 103: 224-9.

4. Pawlikowski M. Expression of follicle stimulating hormone receptors in intra-tumoral vasculature and in tumoral cells- the involve- ment in tumour progression and the perspectives of application in cancer diagnosis and therapy. Endokrynol Pol 2018; 69: 192-8.

5. Casson PR, Lindsay MS, Pisarska MD, Carson SA, Buster JE. Dehydroepiandrosterone supplementation augments ovarian stimulation in poor responders: a case series. Hum Reprod 2000; 15: 2129-32.

6. Barad DH, Gleicher N. Increased oocyte production after treatment with dehydroepiandrosterone. Fertil Steril 2005; 84: 756.

7. Broer SL, van Disseldorp, Broeze J, et al. Added value of ovarian reserve testing on patient characteristics in the prediction of ovarian response and ongoing pregnancy: an individual patient data approach. Hum Reprod Update 2013; 19: 26-36.

8. Broer SL, Dolleman M, van Disseldorp J, et al. Prediction of an excessive response in in vitro fertilization from patient characteristics and ovarian reserve tests and comparison in subgroups: an individual patient data meta-analysis. Fertil Steril 2013; 100: 420-9.

9. Weil S, Vendola K, Zhou J, Bondy CA. Androgen and follicle-stimulating hormone interactions in primate ovarian follicle development. J Clin Endocrinol Metab 1999; 84: 2951-6.

10. Sayyid RK, Evans A, Hersey K, et al. a phase ii, randomized, open-label study of neoadjuvant degarelix versus LHRH agonist in prostate cancer patients prior to radical prostatectomy. Clin Cancer Res 2017; 23: 1974-80.

11. Arce JC, La Marca A, Mirner Klein B, Nyboe Andersen A, Fleming R. Antimullerian hormone in gonadotropin releasing-hormone antagonist cycles: prediction of ovarian response and cumulative treatment outcome in good-prognosis patients. Fertil Steril 2013; 99: 1644-53.

12. Yilmaz N, Uygur D, Inal H, Gorkem U, Cicek N, Mollamahmutoglu L. Dehydroepiandrosterone supplementation improves predictive markers for diminished ovarian reserve: serum AMH, inhibin B and antral follicle count. Eur J Obstet Gynecol Reprod Biol 2013; 169: 257-60.

13. Sullivan SD, Sarrel PM, Nelson ML. Hormone replacement therapy in young women with primary ovarian insufficiency and early menopause Fertil Steril 2016; 106: 1588-99.

14. Qin JC, Fan L, Qin AP. The effect of dehydroepiandrosterone (DHEA) supplementation on women with diminished ovarian reserve (DOR) in IVF cycle: Evidence from a meta-analysis. J Gynecol Obstet Hum Reprod 2017; 46: 1-7.

15. Triantafyllidou O, Sigalos G, Vlahos N. Dehydroepiandrosterone (DHEA) supplementation and IVF outcome in poor responders. Hum Fertil (Camb) 2017; 20: 80-7.

16. Zhang J, Qiu X, Gui Y, Xu Y, Li D, Wang L. Dehydroepiandrosterone improves the ovarian reserve of women with diminished ovarian reserve and is a potential regulator of the immune response in the ovaries. Biosci Trends 2015; 9: 350-9.

17. Hun YC, Hu PH, Wang SY, et al. Subfertility and defective folliculogenesis in female mice lacking androgen receptor Proc Natl Acad Sci USA 2004; 101: 11209-14.

18. Hu Q, Hong $\mathrm{L}, \mathrm{Nie} \mathrm{M}$, et al. The effect of dehydroepiandrosterone supplementation on ovarian response is associated with androgen receptor in diminished ovarian reserve women. J Ovarian Res 2017; 10: 32 .

19. Abide Yayla C, Ozkaya E, Kayatas Eser S, Sanverdi I, Devranoglu B, Kutlu T. Association of basal serum androgen levels with ovarian response and ICSI cycle outcome. Irish J Med Sci 2018; 187: 409-15. 
20. Mamas L, Mamas E. Premature ovarian failure and dehydroepiandrosterone. Fertil Steril 2009; 91: 644-6.

21. Singh N, Zangmo R, Kumar S, et al. A prospective study on role of dehydroepiandrosterone (DHEA) on improving the ovarian reserve markers in infertile patients with poor ovarian reserve. Gynecol Endocrinol 2013; 29: 989-92.

22. Fouany MR, Sharara FI. Is there a role for DHEA supplementation in women with diminished ovarian reserve? J Assist Rreprod Genet 2013; 30: 1239-44.

23. Hassa H, Aydin Y, Ozatik O, Erol K, Ozatik Y. Effects of dehydroepiandrosterone (DHEA) on follicular dynamics in a diminished ovarian reserve in vivo model. Syst Biol Reprod Med 2015; 61: 117-21.

24. Weil S, Vendola K, Zhou J, Bondy CA. Androgen and follicle-stimulating hormone interactions in primate ovarian follicle development J Clin Endocrinol Metab 1999; 84: 2951-6.

25. Nielsen ME, Rasmussen IA, Kristensen SG, et al. In human granulosa cells from small antral follicles, androgen receptor mRNA and androgen levels in follicular fluid correlate with FSH receptor mRNA. Mol Hum Reprod 2011; 17: 63-70.

26. Practice Committee of the American Society for Reproductive Medicine. Comparison of pregnancy rates for poor responders using IVF with mild ovarian stimulation versus conventional IVF: a guideline. Fertil Steril 2018; 109: 993-9.

27. Wang W, Tang Y, Liu H. Protective effects of DHEA and Metformin on the viability of early embryos under oxidative stress are mediated by sustaining mitochondrial biogenesis. Fertil Steril 2015; 104; e146-7.

28. Burger HG. Androgen production in women. Fertil Steril 2002; 77 (Suppl. 4): S3-5.

29. Maggio M, De Vita F, Fisichella A, et al. DHEA and cognitive function in the elderly. J Steroid Biochem Mol Biol 2015; 145C: 281-92.

30. Daan NM, Jaspers L, Koster MP, et al. Androgen levels in women with various forms of ovarian dysfunction: associations with cardiometabolic features. Hum Reprod 2015; 30: 2376-86.

31. Doldi N, Belvisi L, Bassan M, Fusi FM, Ferrari A. Premature ovarian failure: steroid synthesis and autoimmunity. Gynecol Endocrinol 1998; 12: 23-8.

32. Ayesha, Jha V, Goswami D. Premature ovarian failure: an association with autoimmune diseases. J Clin Diagn Res 2016; 10: Qc10qc2.

33. Ott J, Pecnik P, Promberger R, et al. Dehydroepiandrosterone in women with premature ovarian failure and Hashimoto's thyroiditis. Climacteric 2014; 17: 92-6.

34. Syan SK, Minuzzi L, Costescu D, et al. Influence of endogenous estradiol, progesterone, allopregnanolone, and dehydroepiandrosterone sulfate on brain resting state functional connectivity across the menstrual cycle. Fertil Steril 2017; 107: 1246-55.

35. de Moraes-Ruehsen M, Jones GS. Premature ovarian failure. Fertil Steril 1967; 18: 440-61.

36. Atria A. La menopausia precoz y su tratamiento hormonal. Rev Med Chile 1950; 78: 373-7.

37. Soman M, Huang LC, Cai WH, et al. Serum androgen profiles in women with premature ovarian insufficiency: a systematic review and meta-analysis. Menopause 2019; 26: 78-93.

38. Borman E, Check JH, Mitchell-Williams J, Cohen R. No evidence to support the concept that low serum dehydroepiandrosterone (DHEA) sulfate (s) levels are associated with less oocyte produc- tion or lower pregnancy rates. Clin Exp Obstet Gynecol 2012; 39: 429-31.

39. Webber L, Davies M, Anderson R, et al. ESHRE Guideline: management of women with premature ovarian insufficiency. Hum Reprod 2016; 31: 926-37.

40. Qin JC, Fan L, Qin AP. The effect of dehydroepiandrosterone (DHEA) supplementation on women with diminished ovarian reserve (DOR) in IVF cycle: evidence from a meta-analysis. J Gynecol Obstet Hum Reprod 2017; 46: 1-7.

41. Lin LT, Tsui KH, Wang PH. Clinical application of dehydroepiandrosterone in reproduction: a review of the evidence. J Chin Med Assoc2015; 78: 446-53.

42. Tartagni M, De Pergola G, Damiani GR, et al. Potential benefit of dehydroepiandrosterone supplementation for infertile but not poor responder patients in a IVF program. Minerva Ginecol 2015; 67: 7-12.

43. Gleicher N, Ryan E, Weghofer A, Blanco-Mejia S, Barad DH. Miscarriage rates after dehydroepiandrosterone (DHEA) supplementation in women with diminished ovarian reserve: a case control study. Reprod Biol Endocrinol 2009; 7: 108.

44. Lin LT, Wang PH, Wen ZH, et al. The application of dehydroepiandrosterone on improving mitochondrial function and reducing apoptosis of cumulus cells in poor ovarian responders. Int J Med Sci 2017; 14: 585-94.

45. Zhang HH, Xu PY, Wu J, et al. Dehydroepiandrosterone improves follicular fluid bone morphogenetic protein-15 and accumulated embryo score of infertility patients with diminished ovarian reserve undergoing in vitro fertilization: a randomized controlled trial. J Ovar Res 2014; 7: 93.

46. Lin LT, Cheng JT, Wang PH, Li CJ, Tsui KH. Dehydroepiandrosterone as a potential agent to slow down ovarian aging. J Obstet Gynaecol Res 2017; 43: 1855-62.

47. Hu Q, Hong L, Nie M, et al. The effect of dehydroepiandrosterone supplementation on ovarian response is associated with androgen receptor in diminished ovarian reserve women. J Ovarian Res 2017; 10: 32

48. Zhang J, Qiu X, Gui Y, Xu Y, Li D, Wang L. Dehydroepiandrosterone improves the ovarian reserve of women with diminished ovarian reserve and is a potential regulator of the immune response in the ovaries. Biosci Trends 2015; 9: 350-9.

49. Singh N, Zangmo R, Kumar S, et al. A prospective study on role of dehydroepiandrosterone (DHEA) on improving the ovarian reserve markers in infertile patients with poor ovarian reserve. Gynecol Endocrinol 2013; 29: 989-92.

50. Fouany MR, Sharara FI. Is there a role for DHEA supplementation in women with diminished ovarian reserve? J Assist Reprod Genet 2013; 30: 1239-44.

51. Agarwal R, Shruthi R, Radhakrishnan G, Singh A. Evaluation of dehydroepiandrosterone supplementation on diminished ovarian reserve: a randomized, double-blinded, placebo-controlled study. J Obstet Gynaecol India 2017; 67: 137-42.

52. Hassa H, Aydin Y, Ozatik O, Erol K, Ozatik Y. Effects of dehydroepiandrosterone (DHEA) on follicular dynamics in a diminished ovarian reserve in vivo model. Syst Biol Reprod Med 2015; 61: 117-21.

53. Mahmoud YI, Mahmoud AA, Abo-Zeid FS, Fares NH. Effects of dehydroepiandrosterone on the ovarian reserve and pregnancy outcomes in perimenopausal rats (DHEA and fertility in perimenopausal rats). Life Sci 2018; 199: 131-8. 
54. Abide Yayla C, Ozkaya E, Kayatas Eser S, Sanverdi I, Devranoglu B, Kutlu T. Association of basal serum androgen levels with ovarian response and ICSI cycle outcome. Ir J Med Sci 2018; 187: 409-15.

55. Agarwal R, Shruthi R, Radhakrishnan G, Singh A. Evaluation of dehydroepiandrosterone supplementation on diminished ovarian reserve: a randomized, double-blinded, placebo-controlled study. J Obstet Gynaecol India 2017; 67: 137-42.

56. Committee opinion no. 618: Ovarian reserve testing. Obstet Gynecol 2015; 125: 268-73.

57. Abdulkareem AF, Abu-Faza M, Abdelazim IA, Abdelrazek KM, Aboelezz A, Farghali MM. Ultrasound-guided surgical suction evacuation (US-SSE) for missed miscarriage. J Obstet Gynecol Investig 2018; 1: e1-e5. DOI: https://doi.org/10.5114/ jogi.2018.73387 